\title{
The Study of Chromatography Used In Separation Science
}

\author{
Anuj Sharma
}

\section{Back Ground Of Proposed Work}

Chromatography is one of the most commonly used methods to separate molecules of various sizes. The technique is frequently applied to the separation of proteins. Such biotechnical applications require effective tools to predict the chromatographic process in order to avoid tedious and costly laboratory experiments during process development. In this thesis, the use of experiments as well as mathematical models to achieve this goal is demonstrated.

The advantages of using an intrinsic mathematical model for simulation of fixed bed chromatography are demonstrated. The chromatography model includes axial dispersion in the bulk liquid, external and internal mass transfer resistances, and instationary nonlinear adsorption. The intrinsic model is compared to simplified lumped models. The former model is able to describe variations in the physical, kinetic, and operating parameters better than the latter ones. This results in a more reliable prediction of the performance of the chromatography process as well as a better understanding of the underlying mechanisms responsible for the separation

A procedure to determine effective diffusion coefficients of proteins in chromatographic gels, required as model input parameters, is presented. An experimental methodology based on frontal liquid chromatography was combined with a numerical methodology based on a detailed mathematical model describing the chromatographic process including the extra-column dispersion, the dispersion due to the packed bed, the external mass transfer from the bulk phase to the stationary phase, and the diffusive transport within the stationary phase. The procedure has several advantages compared to previously reported methods to determine diffusion coefficients in that no other equipment than an HPLC is required, any class of stationary phases can be investigated as long as the experiments are performed under non-binding conditions, and no modification, e.g., molding of slabs or membranes, to the stationary phase is required. To show the applicability of the procedure, the effective diffusion coefficients of lysozyme, bovine serum albumin, and immunoglobulin $\gamma$ in Sepharose ${ }^{\mathrm{TM}}$ CL-4B were determined and shown to be comparable to those determined by other methods.

Molecularly imprinted polymers (MIPs) are man-made polymeric materials with molecular recognition abilities. They mimic the molecular recognition of naturally occurring molecular recognition elements such as receptors and antibodies by binding target molecules by either non-covalent, covalent, or metal-coordinating interactions.

Traditionally, MIPs are synthesized in the form of monolithic polymers which are subsequently crushed, ground, and sieved to an appropriate size range. In this thesis, a suspension polymerization method to prepare MIPs in the shape of spherical beads is presented. The method involves suspending a prepolymerization solution in mineral oil, used as the continuous phase. The droplets are transformed into solid spherical beads by free-radical polymerization. The beads have been shown to compare well to the traditional irregularly shaped particles prepared from monoliths. The advantages of the method compared to previously reported methods are the low cost and commercial availability of the continuous phase and the absence of the need for stabilizers for the formation of droplets of pre-polymerization solution in the mineral oil. When compared to the method to prepare particles from monolithic polymers, this method is advantageous due to the spherical shape of the resulting beads and the reduction in time needed to prepare a MIP.

When a new MIP is designed, the traditional approach is to use eithera previously reported protocol or rules of thumb based on previous knowledge. This results in non optimized MIPs. The number of possible combinations of monomers, cross-linkers, solvents, and initiators are huge. A full optimization of a MIP formulation therefore requires a large number of experiments. To facilitate the efforts, chemometrics was applied to the work described in this thesis. Three factors (i.e., the amount of monomer, the amount of crosslinker, and the amount of porogen) were chosen as the factors in the model. Multivariate data analysis of the binding to the MIPs indicated how the factors influenced the binding and an optimized MIP composition was identified.

The combined use of the suspension polymerization method to produce spherical beads with the application of chemometrics was shown in this thesis to drastically reduce the number of experiments and the time needed to design and optimize a new MIP. 


\section{The Research Plan}

Physical phenomena, such as diffusion, dispersion, and adsorption/desorption kinetics, along with the set-up of the chromatographic system, govern the outcome of a separation. The diffusion of small molecules in the stagnant film surrounding the stationary phase and within the stationary phase itself is often so fast that it can be neglected. However, in the case of larger molecules, such as polypeptides and proteins, the diffusion is substantially slower and the mass transfer resistances have to be accounted for. The aim of the first part of this work was to design experiments and evaluation procedures to determine diffusion coefficients by frontal chromatographic experiments. To accomplish this, each phenomenon influencing the chromatographic process has to be isolated and evaluated separately. By this approach, modular models taking into account relevant phenomena can be designed, thereby avoiding models based on lumped parameters. This type of intrinsic model is well suited for evaluating the effects of changes in the operating conditions of chromatographic processes, for example, the changes seen at scale-ups. The intrinsic model will be more sensitive to such changes than models involving lumped parameters.

Traditionally the preparation of an optimized molecularly imprinted polymer is a time consuming procedure. The most commonly occurring format to synthesize MIPs in is as bulk polymers that are subsequently crushed, ground and sieved to an appropriate size range. This procedure has to be repeated for each investigated polymer during the optimization. The procedure to crush, ground and sieve bulk polymers result in irregularly shaped particles that perform less good in packed bed applications such as liquid chromatography and solid-phase extraction. The aim with the second half of this thesis was to develop a method for rapid preparation and screening of molecularly imprinted polymer libraries in the shape of spherical beads.

\section{INTRODUCTION}

\section{Content Of Research}

\section{CHROMATOGRAPHY}

Historical background

High and low pressure chromatography

Column chromatography

Positive and negative chromatography

Matrices

Chromatography techniques

\section{MODELING CHROMATOGRAPHY}

Modeling the column

Modeling extra column volumes

Plate height theory

\section{DIFFUSION}

Diffusion in free solution

Diffusion in porous materials

Diffusion of proteins

Predicting diffusion coefficients

Methods to determine diffusion rates

\section{DETERMINATION OF DIFFUSION COEFFICIENTS \\ USING FRONTAL CHROMATOGRAPHY \\ MOLECULAR RECOGNITION \\ THE CONCEPT OF MOLECULAR IMPRINTING}

Non-covalent molecular imprinting

Covalent molecular imprinting

Semi-covalent molecular imprinting

Metal ion mediated molecular imprinting

FORMATS OF MOLECULARLY IMPRINTED

POLYMERS

Irregular particles

Beads

Films and membranes

DESIGN OF MOLECULARLY IMPRINTED

POLYMERS 
Functional monomers

Cross-linking monomers

Porogen

Initiation of polymerization

Optimization of imprinting conditions

CHARACTERIZATION OF MOLECULARLY
IMPRINTED POLYMERS

Methods to study binding

Physical Characterization

Chemical Characterization

APPLICATIONS OF MOLECULARLY IMPRINTED

POLYMERS

Solid-phase extraction

Liquid chromatography

Solid-phase binding assays

Sensors

Synthetic enzymes

\section{Importance Of The Work In Future Aspects}

The number of MIPs needed to be prepared was drastically reduced by the use of chemo metrics. It was shown that the combination of chemo metrics and the novel method to prepare spherical MIP beads is very well suited to rapidly design and optimize a new MIP. I believe that this strategy should be helpful for future developments of well optimized MIPs for various applications. Given that the strategy is easy and rapid there should no longer be any reason to use non optimized protocols for the synthesis of MIPs.

The method to prepare spherical beads is a suspension polymerization method with mineral oil as the continuous phase and the pre-polymerization mixture as the dispersed phase. The suspension is created, without the need of addition of stabilizers, by vigorous mixing with a mixing device. The resulting suspension is stable during the entire polymerization. Recovery of the beads is easily performed by removal of the mineral oil by filtration. The advantages of this method to prepare spherical MIP beads over other methods described in the literature are the low cost and commercial availability of the continuous phase and the absence of the need for stabilizers due to the viscosity of the oil.

In my opinion the future for MIPs looks bright. The industrial need for more and more selective separation methods has increased with time and the demands from regulatory authorities for safer products are not likely to be lowered. Therefore, MIPs should be useful for highly selective chromatographic separations as well as solid-phase extractions for purification and trace enrichment. However, in order to meet these demands and to convince those still not believing in the MIP technology, it becomes more and more vital that the methods to design and synthesize MIPs are improved. Other intriguing applications for MIPs are as sensing elements in sensors, as recognition elements in solid-phase binding assays, and as synthetic enzymes. For all applications of MIPs, it is important that the problems with the 'polyclonality' and the difficulty of applying MIPs in aqueous media are addressed and solved. 